\title{
LOS CUIDADOS A LA DEPENDENCIA EN UN NUEVO HORIZONTE CONSTITUCIONAL PARA CHILE
}

\author{
DEPENDENCY CARE IN A NEW CONSTITUTIONAL HORIZON \\ FOR CHILE
}

MAURICIO MATUS LÓPEZ*

\section{Resumen}

Chile se enfrenta a uno de los mayores desafíos de política socio sanitaria. El envejecimiento de la población demanda nuevos derechos y programas. En este contexto, la discusión constitucional puede ser un punto de encuentro para abordar estos desafíos. El objetivo de este artículo es analizar el diseño e implementación de las políticas de cuidado a la dependencia, con una perspectiva desde la experiencia internacional, para el análisis de la situación nacional. Para ello, se realizó una revisión de la evidencia científica, con énfasis en algunos casos paradigmáticos. Los resultados muestran ciertos consensos en el diseño e implementación de programas públicos, que son particularmente interesantes porque quiebran la lógica tradicional de las políticas de bienestar chileno.

\section{Palabras Claves}

Dependencia, adultos mayores, cuidados de larga duración, políticas públicas.

\begin{abstract}
Chile faces one of the greatest challenges in social and health policy. The ageing population demands new rights and programmes. In this context, the constitutional discussion can be a meeting point to address these challenges.
\end{abstract}


The aim of this article is to analyse the design and implementation of policies on care for dependent adults, from the perspective of international experience, in order to analyse the national situation. To this end, a review of the scientific evidence was carried out, with emphasis on some paradigmatic cases. The results show certain consensus in the design and implementation of public programmes, which are particularly interesting because they break the traditional logic of Chilean welfare policies.

\section{Keywords}

Dependency, older adults, long-term care, public policies.

\section{INTRODUCCIÓN.}

Existe un desfase entre la protección social chilena y su realidad demográfica. El cambio más importante de los próximos años será el incremento de la población adulta mayor y se necesitan nuevas y reformadas políticas para enfrentarlo ${ }^{1}$. Es un fenómeno regional, iniciado a comienzos de siglo, pero que se ha acelerado en la última década ${ }^{2}$. En menos de diez años, en Chile, los mayores de 65 años serán más que los menores de 15 años y en torno a la mitad de este siglo, serán el doble que ellos ${ }^{3}$. No es un escenario probable, es un escenario efectivo. Los adultos mayores del

Artículo recibido para su evaluación el 2 de junio de 2020, y aprobado para su publicación el 2 de julio de 2020

* Doctor en Economía. Universidad Pablo de Olavide. Departamento de Economía, Métodos Cuantitativos e Historia Económica. Sevilla, España. Email: mmatlop@upo.es

1 RODRÍGUEZ, J, RUSSO, M y CARRASCO, M: "Políticas Públicas Para Una Población Que Envejece: Panorama y Propuestas Para El Sistema de Salud Chileno". En Temas de la Agenda Pública, 12(92), 2017. pp. 1-12; GANGA, F et al.: "Rol Del Estado Frente A1 Envejecimiento de La Población: El Caso de Chile". En Convergencia, 22(71), 2016. pp. 175-200; OLIVI, Alessandra, FADDA, G y PIZZI, M "Evaluación de La Calidad de Vida de Los Adultos Mayores En La Ciudad de Valparaíso," Papeles de población, 21(84), 2015. pp. 227-249.

2 HUENCHUAN, S: Envejecimiento, Derechos Humanos y Políticas Públicas. CEPAL, Santiago de Chile, 2009; COTLEAR, D: Population Aging: Is Latin America Ready?. World Bank, Washington D.C., 2011. PELÁEZ, E y MINOLDO, S: "Impacto Del Envejecimiento Sobre Demandas de Servicios En El Cono Sur". En Revista Latinoamericana de Población, 23, 2018. pp. 62-84.

3 UNITED NATIONS: "Revision of World Population Prospects". Disponible en: https:// population.un.org/wpp. Último acceso el 15 de julio de 2020. 
futuro son los adultos de la actualidad. Ya nacieron y son los padres de la generación actual de estudiantes.

Una parte de estos mayores, por motivos de salud o simple deterioro físico y cognitivo, no serán capaces de desarrollar una vida autónoma. Necesitarán ayuda para vestirse, alimentarse, bañarse, etc. En la actualidad, estos cuidados recaen principalmente en las familias y concretamente, en las mujeres. Hijas, madres y esposas desarrollan un trabajo generador de valor que no se encuentra remunerado y que carece del reconocimiento como tal ${ }^{4}$.

La legislación chilena sigue asociando preferentemente el cuidado a la infancia, y avanza más lentamente en la extensión de la protección a los dependientes mayores. ${ }^{5} \mathrm{La}$ oportunidad de crear un nuevo cuerpo constitucional, surgido de un proceso democrático, que reemplace a aquel nacido en dictadura, parece de necesidad evidente. Es la oportunidad de pensar en el largo plazo y ampliar la protección a quienes lo necesitan y, sobre todo, a quienes lo van a necesitar en un futuro cercano.

El objetivo de este artículo es analizar el diseño e implementación de políticas de cuidado a la dependencia, con una perspectiva desde la experiencia internacional, para el análisis de la situación nacional. Se pretende aportar a la discusión sobre cuidados en nuevo marco normativo. Para esto, se presenta una discusión basada en la evidencia científica internacional sobre protección de las personas dependientes.

\section{EL CAMBIO DEMOGRÁFICO Y LA SITUACIÓN DE DEPENDENCIA EN CHILE}

Cuando se habla de dependencia o cuidados de larga duración (longterm care) se está haciendo referencia a la situación en la que una persona necesita ayuda de terceros para realizar las actividades de la vida diaria,

4 GONZÁlVEZ, H: "Género, Cuidados y Vejez: Mujeres en El Medio Del Trabajo Remunerado y Del Trabajo de Cuidado En Santiago de Chile". En Prisma Social: revista de investigación social, 21, 2018. pp. 194-218; BARRIGA, F et al.: No Es Amor, Es Trabajo No Pagado Un Análisis Del Trabajo de Las Mujeres En El Chile Actual. Fundación Sol, Santiago de Chile, 2020.

5 MATUS-LÓPEZ, M y CID, C: "Building Long-Term Care Policies in Latin America: New Programs in Chile". En Journal of the American Medical Directors Association, 16(10), 2015. p. 900.e7-900.e10. 
por un largo periodo de tiempo ${ }^{6}$. Son estos tres conceptos los que definen esta situación.

Se diferencia de la discapacidad en que esta última, no necesariamente está vinculada a la necesidad de ayuda. Una persona con una discapacidad auditiva puede llevar una vida totalmente independiente, con los medios tecnológicos adecuados. Además, la dependencia afecta más a las personas que, debido al envejecimiento natural, han perdido la capacidad de vivir de forma independiente.

Los factores que determinan la demanda de estos servicios han sido ampliamente estudiados ${ }^{7}$, Existe consenso en destacar dos como los más importantes. El primero es el cambio demográfico hacia un aumento de la proporción de personas mayores ${ }^{8}$. Esto, porque el número de personas que requieren ayuda se multiplica a partir de los 65 años y se acelera todavía más, después de los 80 años de edad. La prevalencia se multiplica por 3,5 a partir de los 65 años y por 18 a partir de los 80 años $^{9}$. El segundo, es el

6 WHO: A Glossary of Terms for Community Health Care and Services for Older Persons, World Health Organization, Kobe, 2004; EUROPEAN COMMISSION: "The 2015 Ageing Report: Economic and Budgetary Projections for the 28 EU Member States (2013-2060)". En European Economy, 3, 2015; OECD, Health at a Glance 2013,OECD Publishing, Paris, 2013. La mayor parte de la bibliografía científica internacional se encuentra indexada al concepto de cuidados de larga duración en inglés: long-term care. La utilización del término dependencia, en este sentido, se remite principalmente a los casos hispanohablantes de España y América Latina.

7 MAISONNEUVE, C De la y OLIVEIRA, J: A Projection Method for Public Health and Long-Term Care Expenditures, Working Papers OECD Economics Department, 1048, 2013; COLOMBO, Francesca et al.: Help Wanted? Providing and Paying for Long-Term Care, OECD Publishing, Paris, 2011; VERBEEK-OUDIJK, Debbie et al., Who Cares in Europe? A Comparison of Long-Term Care for the over-50's in Sixteen European Countries, Netherlands Institute for Social Research, The Hague, 2014; DE MEIJER, Claudine et al.: "Determinants of Long-Term Care Spending: Age, Time to Death or Disability?". En Journal of Health Economics, 30(2), 2011. pp. 425-438; WU, Chen-Yi et al.: "Determinants of Long-Term Care Services among the Elderly: A PopulationBased Study in Taiwan". En PLoS ONE, 9(2), 2014. p. e89213.

8 Algunos autores señalan que no es la edad la que determina la demanda, sino que, al construir modelos explicativos, se ajusta mejor la variable "tiempo de vida restante" (time to death). Este concepto se conoce como red herring y existe abundante discusión sobre el mismo en la utilización de servicios de salud COSTA-FONT, Joan, y VILAPLANAPRIETO, Cristina: "More than One Red Herring'? Heterogeneous Effects of Ageing on Health Care Utilisation". En Health Economics, (forthcoming), 2020.

9 En comparación con el tramo 45-49 años de edad. Ambos sexos. VILAPLANA, Cristina: "Estimation of Dependency Situations in Spain Using the EDAD 2008". En Hacienda Pública Española, 194, 2010. pp. 125-175. 
impacto de género de la inexistencia de estas políticas ${ }^{10}$. Ante la ausencia, el cuidado recae principalmente en las mujeres, que asumen este trabajo sin retribución económica y con una intensidad que les dificulta participar en el mercado laboral.

En Chile, el envejecimiento es un hecho. Hace solo treinta años, las personas mayores de 65 años eran el 5,9\% de la población total. En menos dos décadas superará el 20\%. Esto implica que para 2030 habrá en el país unos 3,35 millones de personas de 65 y más años. Es decir, más adultos mayores que menores de 15 años.

En 2009, el SENAMA realizó una Encuesta a las Personas Mayores, que permitió estimar cuántas de ellas necesitaban ayuda para realizar actividades de la vida diaria ${ }^{11}$. La definición de dependencia que se utilizó es algo más compleja que la internacional, pero se basa en las mismas variables; capacidad de realizar actividades básicas (alimentarse, desplazarse, bañarse, etc.) y actividades instrumentales (ir de compras, vida social, etc...). Los resultados permitieron estimar que el $24 \%$ de los mayores de 60 años tenían algún grado de dependencia y que más de la mitad de ellos, estaba en una situación grave. A este estudio le han seguido otros. Utilizando la misma definición, pero la encuesta CASEN de 2015, se estimó que el 3,5\% de la población sufría dependencia, y que este porcentaje se elevaba al $18 \%$ de los mayores de 65 años $^{12}$. Con la misma encuesta, pero para el año 2017, el Ministerio de Desarrollo Social llegó a una cifra algo menor, situando en $14,4 \%$ la tasa de dependencia entre personas de 60 y más años ${ }^{13}$. Una tercera fuente utilizada es la Encuesta de Discapacidad 2015. De acuerdo a ella, la dependencia se situaría en el $21,8 \%$ de los mayores de 60 años de edad ${ }^{14}$.

10 MATUS-LÓPEZ, M y RODRÍGUEZ-MODROÑO, P: "Presiones de Oferta y Demanda Sobre Políticas Formales de Cuidados En Latinoamérica". En Reforma y Democracia, 60, 201. pp. 103-130.

11 GONZÁLEZ, F, MASSAD, C y LAVANDEROS, F: Estudio Nacional de La Dependencia En Las Personas Mayores, Servicio Nacional del Adulto Mayor, Santiago de Chile, 2010.

12 VILlalobOS, P: "Panorama de La Dependencia En Chile: Avances y Desafíos" Revista Medica De Chile, 147(1), 2019. pp. 83-90.

13 MINISTERIO DE DESARROLLO SOCIAL: Adultos Mayores. Síntesis de Resultados. CASEN 2017, MIDESO, Santiago, 2017. Disponible en: http://observatorio. ministeriodesarrollosocial.gob.cl/casen-multidimensional/casen/docs/Resultados Adulto_Mayores_casen_2017.pdf Acceso el 20 de julio de 2020.

14 ARCE, P et al.: Caracterización de La Dependencia En Las Personas En Situación de Discapacidad a Partir Del II Estudio Nacional de La Discapacidad, Servicio Nacional de la Discapacidad, Santiago, 2017. 
Si se consideran estos cuatro estudios y se homogeneizan por tramo de edad, se tiene que entre el 17,9\% y el $28,9 \%$ de las personas mayores de 65 años son dependientes en Chile. Esto equivaldrá a un rango de 600 mil a casi 1 millón de personas en 2030.

Existen dos estimaciones de cuánto costaría un sistema de atención a la dependencia para Chile. Los trabajos están relacionados en sus fuentes, pero difieren en sus metodologías. Estiman el costo total entre $1.214 \mathrm{y}$ 3.096 millones de dólares. Es decir, un esfuerzo de entre 0,45\% y 1,04\% del PIB $^{15}$.

En el país no existe una política nacional de atención a la dependencia. El modelo no se ha modificado en los últimos años. Este descansa casi exclusivamente en el cuidado en los hogares, servicios vinculados a agencias religiosas y programas fragmentados de financiamiento público y gestión local ${ }^{16}$.

A nivel latinoamericano, solo Uruguay tiene un sistema de cuidados de alcance nacional ${ }^{17}$. Costa Rica se encuentra avanzando en uno y la mayoría de los países de niveles de envejecimiento similar, están en la etapa de estimar la demanda potencial o en la discusión sobre la necesidad de implementar un modelo sostenible ${ }^{18}$.

15 CID, C. dir.: Análisis Prospectivo de Un Seguro Obligatorio de Dependencia Para Adultos Mayores. Informe Final. CIEDESS, Santiago de Chile, 2014; MATUS-LÓPEZ, $\mathrm{M}$, and CID, C: "Costo de un sistema de atención de adultos mayores dependientes en Chile, 2012 - 2020". En Pan American Journal of Public Health, 36(1), 2014. Pp. 31-36. El rango menor corresponde a Matus-López \& Cid (2014) y el rango mayor corresponde a Cid dir. (2014).

16 MATUS-LÓPEZ, M y CID, 2014. ob.cit.; VILLALOBOS, P, 2019. ob.cit; VILLALOBOS, P: "Long-Term Care Systems as Social Security: The Case of Chile". En Health Policy and Planning, 33(9), 2018. pp. 1018-1025.

17 MATUS-LÓPEZ, M y CID, C: "New Long-Term Care Policies in Latin America: The National System of Care in Uruguay". En Journal of the American Medical Directors Association, 17(7), 2016. pp. 663-665; ARANCO, Natalia: Panorama de Envejecimiento y Atención a La Dependencia: Resumen Uruguay. BID, Washington, D.C., 2019.

18 MEDELLIN, N, JARA, P y MATUS-LOPEZ, M: "Envejecimiento y Atención a La Dependencia En Costa Rica," Nota Técnica IDB-TN-1820, 2019; JARA MALEŠ, P, MATUS-LÓPEZ, M y CHAVERRI-CARVAJAL, A: "Tendencias y Desafíos Para Conformar Un Sistema de Cuidados de Larga Duración En Costa Rica," Nota Tecnica IDB_TN 1878, 2020; NORORI, M “Addressing the 'Tsunami' of Long-Term Care Needs in Latin America: Is Preparation Feasible?". En Journal of the American Medical Directors Association, 19(9), 2018. pp. 731-732. 


\section{EL DERECHO A SER CUIDADO. CHILE Y LOS EJEMPLOS INTERNACIONALES DE PROTECCIÓN}

Cuando se analiza la transición chilena, llama la atención de que la Constitución aprobada durante la dictadura no hubiera sido reemplazada por una nueva, democrática. Las razones han sido ampliamente discutidas y las explicaciones son las mismas que tratan de explicar la continuidad de lo medular del modelo social y económico de Pinochet ${ }^{19}$.

La posibilidad de configurar una nueva carta de principios fundamentales, en un contexto democrático y con amplia participación social, abre la oportunidad de incluir en ella nuevos derechos.

En la actualidad, el país no cuenta con un reconocimiento constitucional explícito de los derechos de los adultos mayores y menos aún, de los mayores dependientes. Solo son aplicables, de forma indirecta, algunas protecciones a grupos más amplios ${ }^{20}$.

En este sentido, la experiencia internacional es muy variada. Las constituciones nacionales, en función de su temporalidad, incluyen ciertos derechos de salud y atención médica y su expresión se hace más o menos aplicable a los cuidados de los mayores y menos, a las personas dependientes ${ }^{21}$.

En el caso de los países europeos con sistemas de atención a la dependencia, la declaración de los principios fundamentales suele

19 Las explicaciones más referidas apuntan a a las restricciones legales y políticas que la dictadura dejó al gobierno. Ver en BOENINGER, E: Democracia En Chile. Lecciones Para La Gobernabilidad. Andrés Bello, Santiago de Chile, 1998. p. 371. JOCELYNHOLT, A: El Chile Perplejo. Del Avanzar Sin Transar Al Transar Sin Parar. Planeta, Santiago de Chile, 1999, Pág. 260. Pero también a un viraje ideológico de la propia Concertación gobernante. Ver en MOULIAN, T: Chile Actual. Anatomía de Un Mito. LOM: Santiago de Chile, 1998. p. 361; CADEMARTORI, J: Chile. El Modelo Neoliberal. CESOC, Santiago de Chile, 1998. p. 23; GONZÁLEZ, J: El Efecto de La Ambigüedad En La Transición Chilena, RIL Editores, Santiago de Chile, 2008. p. 40.

20 FINSCHI, A: "Adulto Mayor y El Derecho Chileno. Estado Actual En Chile, Análisis Jurídico Comparado y Observaciones a La Convención Interamericana de Derechos Humanos de Las Personas Mayores" En: Revista Familia y Derecho, 1, 2018. pp. 3554; LATHROP, Fabiola: "Protección Jurídica de Los Adultos Mayores En Chile”. En: Revista Chilena de Derecho 36 (1), 2009. pp. 77-113.

21 KINNEY E y CLARK, B "Provisions for Health and Health Care in the Constitutions of the Countries of the World". en: Cornell International Law Journal, 37(2), 2004; MARTÍNEZ, A: "La Protección Jurídica de Las Personas Mayores Desde La Perspectiva de Los Derechos Humanos”. En: Revista de Derecho de la UNED, 17, 2015. pp. 1067-1102. 
ser extensiva en derechos, regulando incluso el deber de velar por la distribución de los bienes materiales.

En el marco de la convergencia de derechos, el Tratado de la Unión Europea, en su artículo 3 señala como objetivo de la Unión, el bienestar de la población. En el desarrollo de este deber, la Comisión lanzó, en 2016, el proceso de construcción de los Pilares de los Derechos Sociales de la UE, aprobado un año más tarde con un listado de 20 derechos $^{22}$. El número $18^{\circ}$ trata los cuidados de dependencia, estableciendo que toda persona tiene derecho a cuidados de larga duración asequibles y de buena calidad, en particular, de asistencia a domicilio y servicios comunitarios ${ }^{23}$.

Una vez establecido el derecho en su forma más extensiva, o de manera explícita al cuidado, el desarrollo legal configura las diferencias entre un modelo y otro.

Para comprender estos modelos es necesario remitirse a las leyes y decretos que los regulan, y en este sentido, la comparación nunca es lineal. A modo de ejemplo, se presentan tres paradigmas actuales.

\section{El modelo extensivo; Países Bajos.}

Existe consenso que uno de los modelos más completos es el de los Países Bajos. Con un costo entre 3,5\% y $4 \%$ del PIB y una cobertura cercana al $12 \%$ de los adultos mayores, es uno de los más extensivos ${ }^{24}$. Se declara universal y no discrimina en el acceso. Fue uno de los primeros en ser desarrollados. Su origen se encuentra en el seguro público para gastos médicos excepcionales de $1968^{25}$. A partir de entonces ha tenido una serie de transformaciones, principalmente durante la década de los años 1990s. El objetivo de estas modificaciones fue la contención de costos y se llevó a cabo a través de la introducción de herramientas de la nueva gestión pública

22 EUROPEAN COMMISSION: European Pillar of Social Rights. EC, Bruselas, 2017.

$2318^{\circ}$. "Long-term care. Everyone has the right to affordable long-term care services of good quality, in particular home-care and community-based services".

24 OECD: Health at a Glance 2019. OECD Publishing, Paris, 2019; EUROPEAN COMMISSION, "The 2018 Ageing Report: Economic and Budgetary Projections for the 28 EU Member States (2016-2070)" En Institutional Paper, 79, 2018. Pág. 406; CENTRUM INDICATIESTELLING ZORG, “Feiten En Cijfers” Disponible en: https:// www.ciz.nl/over-ciz/feiten-en-cijfers Acceso el 25 de julio de 2020.

25 SCHUT, F y VAN DEN BERG, B: "Long-Term Care Insurance in the Netherlands". En Costa-Font, J. y Courbage, C. (Eds.): Financing Long-Term Care in Europe: Institutions, Markets and Models. Palgrave Macmillan, Hapshire, 2011. pp. 103-124. 
y el fomento de la asistencia domiciliaria en lugar de la residencial ${ }^{26}$. En 2015 se realizó la última reforma de calado. Se reestructuraron las fuentes de financiamiento en tres marcos legales. Uno destinado a los cuidados residenciales, a cargo de las oficinas regionales de cuidados, otra centrada principalmente en la atención domiciliaria y personal, a cargo del seguro público de salud, y una tercera, para otros servicios residenciales, a cargo de los gobiernos locales ${ }^{27}$. Por ello, la coordinación de políticas públicas es esencial en el modelo neerlandés. Como resultado de esta estructura, el financiamiento se nutre de cotizaciones de las personas trabajadoras, de impuestos generales y de copagos de las familias ${ }^{28}$.

\section{El caso tardío; España.}

Un segundo caso relevante es el de España. Con un costo estimado entre $0,75 \%$ y $0,9 \%$ del PIB y una cobertura del $8,6 \%$ de los adultos mayores, ofrece amplia cobertura con bajo gasto $^{29}$. A diferencia de los países centro europeos, desarrolló su sistema de atención a la dependencia de forma más tardía; entre 2007 y $2010^{30}$. La Ley 39/2006, estableció el sistema nacional y hace referencia, en su exposición de motivos, a los artículos $49^{\circ}$ y $50^{\circ}$ de la Constitución Española, sobre derechos de las personas mayores y de las personas con discapacidad. Al igual que los Países Bajos, el sistema español se declara de acceso universal y público. $\mathrm{Su}$ financiamiento se basa en impuestos generales, con aportaciones de los recursos del gobierno central y de los gobiernos autonómicos, no

26 DA ROIT, B: "Long-Term Care Reforms in the Netherlands". En: Ranci, Costanzo y Pavolini, Emmanuele: Reforms in Long-Term Care Policies in Europe. Springer, New York, 2013. pp. 97-115.

27 MAARSE, J y JEURISSEN, P: "The Policy and Politics of the 2015 Long-Term Care Reform in the Netherlands". En Health policy, 120(3), 2016. pp. 241-245. Ley de Cuidados de Larga duración (WLZ: Wet Langdurige Zorg), Ley de Seguro Social (ZVW: Zorgverzekeringswet) y Ley de Seguro Social (WMO: Wet Maatschappelijke Ondersteuning), respectivamente.

28 DIJKHOFF, T: "Long-Term Care in the Netherlands". En: Becker, Ulrich y Reinhard, Hans-Joachim: Long-Term Care in Europe: A Juridical Approach: Springer International Publishing, Cham, 2018. pp. 309-352.

29 OECD, 2019, cit.; EUROPEAN COMMISSION, 2018. Op cit.; MINISTERIO DE SANIDAD CONSUMO Y BIENESTAR SOCIAL: Estadística Mensual SAAD, Disponible en: https://www.imserso.es/imserso_01/documentacion/estadisticas/info_d/ estadisticas/est_inf/inf_gp/index.htm. Último acceso el 16 de julio de 2020.

30 PENA-LONGOBARDO, L et al.: "The Spanish Long-Term Care System in Transition: Ten Years since the 2006 Dependency Act”. En Health Policy, 120(10), 2016. pp. 1177-1182. 
incluyendo cotizaciones al salario. Considera copagos, pero estos varían según cada administración regional ${ }^{31}$.

\section{El avance latinoamericano; Uruguay.}

El tercer caso relevante es el de Uruguay. El único país latinoamericano que ha implementado un sistema integral ${ }^{32}$. Este se aprobó a través de la Ley $N^{\circ} 19353$ de 2015 y creó un Sistema Nacional de Cuidados (SNC), para menores, personas con discapacidad, dependientes y cuidadoras ${ }^{33}$. La ley hace mención explícita a la Constitución de la República en materia de las atribuciones del poder ejecutivo para organizar el sistema. En parte, porque la Constitución, de 1967, no tiene artículos específicos sobre discapacidad o adultos mayores. La mayor cercanía se produce en los derechos de salud. Establece el deber del Estado de legislar en esta materia y, curiosamente, el deber de los ciudadanos de cuidarse a sí mismos (Art. 44).

La Ley del SNC establece la universalidad en el acceso a los servicios y contempla explícitamente la complementariedad pública y privada en su desarrollo. Siguiendo la lógica del modelo español, cuenta con un baremo de valoración de la severidad, que permite priorizar y asignar recursos de acuerdo a la disponibilidad de medios ${ }^{34}$. Asimismo, también se financia únicamente con impuestos generales y considera copagos de los usuarios. La parte del SNC que corresponde a servicios de atención a la dependencia, comenzó a implementarse algo más tardíamente, ya que el SNC priorizó en el desarrollo de servicios a la infancia ${ }^{35}$.

\section{LOS MODELOS DE ATENCIÓN}

Cada una de las ordenaciones específicas de los países, da origen a modelos

31 ASOCIACIÓN ESTATAL DE DIRECTORES Y GERENTES DE SERVICIOS SOCIALES: XX Dictamen Del Observatorio de La Ley 39/2006 de Promoción de La Autonomía Personal y Atención a Las Personas En Situación de Dependencia, AEDRSS, Madrid, 2020.

32 ARANCO, cit.

33 MINISTERIO DE DESARROLLO SOCIAL DE URUGUAY: "Sistema Nacional de Cuidados". Disponible en: https://www.gub.uy/sistema-cuidados. Último acceso el 17 de julio de 2020. .

34 MATUS-LÓPEZ Y CID, cit.

35 CARUSO, M, GALIANI, S e IBARRARAN, P: "Long-Term Care in Latin America and the Caribbean: Theory and Policy Considerations" En Economia-Journal of the Latin American and Caribbean Economic Association, 20(1), 2019. p. 1-32. 
con sus particularidades. La existencia de una política de atención a la dependencia no cierra la discusión. No se trata de cumplir con una ayuda, sino que efectivamente se solucionen los problemas del cuidado y se responda de una forma eficiente y sostenible a la mejora de las condiciones de vida de las personas.

Cuando se habla de servicios propiamente tal, dos son los principales. El primero y más tradicional es la atención en residencias. Establecimientos de ambiente doméstico que parveen atención médica y de cuidados, de día y de noche, a personas en condiciones de dependencia muy severas que deben trasladarse a residir de forma permanente a un centro especializado ${ }^{36}$. Estos centros cumplen con estándares de cualificación y calidad, que les diferencia de los comúnmente conocidos como hogares de ancianos. Aunque pueden compartir establecimiento físico con éstos, constan de personal cualificado e integración con el sistema de salud, que permite hacer un seguimiento de la evolución del estado físico, mental y emocional de la persona dependiente. Por ello, la normativa, la fiscalización y la financiación, son tan importantes. Son centros caros, cuyo coste por residente es aproximadamente dos veces el ingreso mediano de los jubilados ${ }^{37}$. Es esta cualidad la que lleva a que la mayoría de los países organicen el financiamiento de forma solidaria o con riesgo compartido ${ }^{38}$. Los fondos suelen configurarse como los diseñados para las enfermedades catastróficas. Es decir, con aportes individuales a un fondo común o directamente con impuestos generales.

El segundo servicio más común es la asistencia en el domicilio. Existen varios tipos, pero la más extendida consiste la visita al hogar del dependiente, de una persona formada en cuidados y con nociones básicas o intermedias de medicina primaria y/o geriátrica. El número de horas se establece mensual o semanal y depende de la severidad del estado de salud. La duración de una visita varía bastante, pero los máximos se sitúan en torno a tres horas diarias ${ }^{39}$.

Esta visita es fundamental porque genera información compartida con el sistema sanitario, que permite el control continuo y la identificación de situaciones de riesgo que alerten la intervención o al traslado a un centro

36 SANFORD, A et al., "An International Definition for 'Nursing Home". En: Journal of the American Medical Directors Association 16(3), 2015. pp. 181-184.

37 OECD, 2019., cit.

38 COSTA-FONT, J, COURBAGE, C y SWARTZ, K: "Financing Long-Term Care: Ex Ante, Ex Post or Both?" En Health Economics, 24(51), 2015. pp. 4-57.

39 En Francia, por ejemplo, en el grado más grave se estiman unas 2,1 horas al día (63 al mes), en España existe un máximo de 70 horas al mes y en Uruguay, de 80 horas. 
residencial o al sistema de salud ${ }^{40}$. Es importante señalar que las tareas de cuidado pueden o no incluir algunos servicios de tareas domésticas como limpiar o cocinar. Sin embargo, su objetivo central son las actividades relacionadas directamente con las actividades de la vida diaria. En los modelos más desarrollados se incluyen, además de la asistencia para el cuidado, servicios de distribución de comidas preparadas y asistencia en tareas domésticas, de forma separada.

Otros servicios, complementarios o independientes, son los centros de día y/o noche, la teleasistencia, mejoras en el hogar que faciliten la movilidad y campañas de promoción de la autonomía, entre otros ${ }^{41}$.

En la última década del siglo pasado, adquirió fuerza una corriente reformista que buscó dar más capacidad de decisión a las personas beneficiarias ${ }^{42}$. Una de las herramientas impulsadas, en los cuidados de larga duración, fueron las transferencias monetarias (cash-for-care). La fundamentación fue transformar el derecho al servicio (plaza residencial $o$ asistencia en el domicilio), en el derecho a decidir el servicio. Es decir, a percibir una cantidad de dinero que le permita a la propia beneficiaria decidir el qué se adecúe mejor a sus preferencias.

Sin embargo, las transferencias sólo comparten el nombre y son muy distintas en cada modelo ${ }^{43}$. En Italia, la Indennità di Accompagnamento alcanza los 520 euros al mes y no está condicionada al control sobre el uso del dinero ${ }^{44}$. En Francia, la Allocation Personnalisée à l'Autonomie llega hasta los 1.700 euros al mes, no se puede contratar a la esposa/a y se debe

40 ANTUNES, V y MOREIRA, J “Approaches to Developing Integrated Care in Europe: A Systematic Literature Review" En: Journal of Management \& Marketing in Healthcare, 4(2), 2011. pp. 129-135.

41 Para un ejemplo de la amplitud de servicios, ver MAARSE y JEURISSEN, cit.

42 WEBSTER, CH: The National Health Service: A Political History, Oxford University Press, Oxford, 1998.

43 DA ROIT, B y LE BIHAN, B: "Similar and Yet So Different: Cash-for-Care in Six European Countries' Long-Term Care Policies". En: The Milbank Quarterly, 88(3), 2010. pp. 286-309; DA ROIT, B y GORI, Cristiano: "The Transformation of Cash for care Schemes in European Long term Care Policies” En: Social Policy \& Administration, 53 (4), 2019. pp. 515-518.

44 HOHNERLEIN, E: "Long-Term Care Benefits and Services in Italy". En: Becker, Ulrich y Reinhard, Hans-Joachim: Long-Term Care in Europe: A Juridical Approach: Springer International Publishing, Cham, 2018. pp. 229-307. Istituto Nazionale Previdenza Sociale, Disponible en: https://www.inps.it/NuovoportaleINPS/default. aspx?itemdir=50194\&lang=IT 
rendir cuenta del uso de los fondos ${ }^{45}$. En Países Bajos, la Persoonsgebonden Budget se establece en un valor cercano a dos terceras partes del costo de los servicios, hasta unos 3.600 euros al mes, se controla su uso y está orientado a la contratación de servicios formales ${ }^{46}$. La extensión, beneficios e impacto de cada una de estas tres prestaciones es muy diferente en términos de creación de empleo, calidad del cuidado y sesgo de género.

En la realidad, por lo tanto, los modelos de prestaciones difieren bastante. Entre los analizados en el apartado anterior, el de los Países Bajos es uno de los más generosos, pero también uno de los más caros. Favorece los servicios de atención directos y aunque incluye la posibilidad de recibir una prestación monetaria (en lugar del servicio), esta opción ha sido escasamente preferida por los usuarios. En 2017, aproximadamente el 83\% de las prestaciones fueron en especie, un $14 \%$ en prestación económica y otro 3\% una combinación de ambas modalidades ${ }^{47}$.

Por su parte, el modelo español ofrece una canasta de servicios residenciales y domiciliarios, y prestaciones económicas. Hasta 2014, las transferencias monetarias para cuidados en el entorno familiar representaron más del $40 \%$ de los beneficios, comenzando a reducirse únicamente a partir de entonces. En la actualidad, estas prestaciones son del 31,3\%, mientras que los servicios directos de atención suman el $35,1 \%{ }^{48}$. La tendencia tiene relación con la fuerte crisis que sufrió el país entre 2008 y 2012, que fue acompañada de políticas de austeridad que redujeron el gasto público y generaron incentivos en favor de las transferencias monetarias ${ }^{49}$.

45 DA ROIT, B y LE BIHAN, B., cit. El montante se cifra según severidad, ingreso y tipo de beneficio (domiciliario o residencial). El máximo corresponde a mayor severidad, menor ingreso y residencial. Service-Public Disponible en: https://www.service-public. $\mathrm{fr} /$ particuliers/vosdroits/F10009

46 DIJKHOFF, cit. Long-term Care Monitor. Persons with PGB; budget spending, care package. Disponible en: https://www.monitorlangdurigezorg.nl/kerncijfers/gebruik/ gebruik-persoonsgebonden-budget

47 KELDERS, Y y DE VAAN, K: ESPN Thematic Report on Challenges in Long-Term Care. Netherlands. European Commission, Bruselas, 2018.

48 Se incluyen en servicios directos la asistencia en el domicilio, la residencial y los centros de día y noche. El resto de beneficios son dos prestaciones económicas para la contratación de servicios externos con el 11,5\%, la teleasistencia con 17,6\% y acciones de promoción con 4,5\%. MINISTERIO DE SANIDAD CONSUMO Y BIENESTAR SOCIAL. 2019, cit.

49 MATUS-LÓPEZ, M, and RAPUN, M: "Los Cuidados Para La Atención a La Dependencia." En Gálvez, Lina dir.: La Economía de Los Cuidados. DeCulturas, Sevilla, 2016. pp. 163-186. 
Por último, Uruguay ha comenzado recientemente a extender sus servicios de cuidados para mayores dependientes. En la actualidad, la ofrece asistencia en el domicilio a 6.125 personas $^{50}$. Funciona como un voucher similar al francés, pero con intermediación directa de la administración, para dar de alta en la seguridad social a los cuidadores. Cuenta, además con centros de día y teleasistencia. Las plazas de atención residencial, no obstante, no está entre los servicios ofrecidos. Existe un programa bajo responsabilidad del Banco de Previsión Social, pero no tiene vinculación directa con dependencia ${ }^{51}$.

\section{CONCLUSIONES}

Todo parece indicar que América Latina y Chile en particular, se acercan a un escenario de implementación de políticas de cuidados a personas dependientes ${ }^{52}$. No es una opción, sino una necesidad. No solo en términos morales y de responsabilidad política, sino también en términos económicos.

Ningún país que quiera seguir creciendo económicamente, puede mantener el trabajo de cuidados en el ámbito informal. La productividad de estos trabajos es baja y mina los resultados de las inversiones que se hacen en educación y salud.

La ventaja que tienen los países que ahora enfrentan esta situación, es que existen cerca de cuarenta países que ya cuentan con políticas de atención a la dependencia, y cientos de estudios que han evaluado su funcionamiento y su impacto durante más de treinta años. Gracias a ello, un nuevo marco constitucional podría incorporar la protección de la población dependiente, basada en la frontera del conocimiento social y económico.

Al respecto, y vista la evidencia científica internacional, hay tres cuestiones que cabría destacar y tener en consideración en este proceso.

Seguramente lo más importante de esta discusión es que propicia el quiebre conceptual de los lineamientos generales que han guiado la

50 MINISTERIO DE DESARROLLO SOCIAL DE URUGUAY, 2020, cit.

51 El programa cupo-cama está orientado principalmente como solución habitacional para mayores sin recursos. Banco de Previsional Social Disponible en: https://www.bps.gub. uy/10668/cupo-cama.html

52 PELÁEZ, E y MINOLDO, S. 2018. op. cit.; MATUS-LÓPEZ, M: “Thinking about LongTerm Care Policies for Latin America”. En Salud Colectiva, 11(4), 2015, Pág: 485-496; ARANCO, N: "Panorama de Envejecimiento y Atención a La Dependencia: Estado de Salud de América Latina y El Caribe” En: Resumen de Políticas IDB-PB 273, 2018. 
política social chilena en casi toda su historia y se posiciona frontalmente en contra de la lógica establecida desde la dictadura y la transición. Se trata de cambiar el paradigma de los programas sociales focalizados en los más pobres y de las políticas de salud segmentadas por capacidad de pago y riesgo de gasto ${ }^{53}$. En definitiva, de concebir políticas de carácter nacional para todo el conjunto de la población, se acuerdo al paradigma de los Estados de Bienestar más desarrollados. Desde hace muchos años que la evidencia internacional ha concluido que las políticas de dependencia deben buscar ser universalistas, solidarias y de responsabilidad pública ${ }^{54}$. No es solo una cuestión de principios y preferencias sociales, sino que es una exigencia de eficiencia financiera.

El costo de los servicios necesarios para una persona en situación de dependencia, supera los ingresos per cápita de la gran mayoría de la población. Muchos hogares no pueden hacer frente a la contratación de servicios de cuidados sin empobrecerse. Como, además, no es posible prever con relativa certeza, quiénes de los adultos jóvenes actuales sufrirá dependencia en su futuro, tampoco funciona de forma extensiva el ahorro individual ${ }^{55}$. Los seguros privados han mostrado que, en esta situación, sufren demasiados fallos de mercado, que fracasan en su intento de ser una respuesta efectiva ${ }^{56}$. Los costos de la dependencia se comportan como costos catastróficos, similar al de los de enfermedades raras, y demandan fondos mancomunados con mecanismos de solidaridad de ingresos. Es decir, se trata de una política de protección social universal, con una lógica distinta a la que rige actualmente. Un cambio conceptual más profundo que invita a pensar en políticas iguales para todos.

53 Sobre políticas focalizadas ver SOTTOLI, S "La Política Social En América Latina Bajo El Signo de La Economía de Mercado y La Democracia” En: European Review of Latin American and Caribbean Studies, 68, 2000. pp. 3-22. Sobre políticas de salud ver MONTOYA, C: La Salud Dividida. Chile 1990-2010, CEIBO Ediciones: Santiago de Chile, 2013.

54 COMAS-HERRERA, A, WITTENBERG, R y PICKARD, L:"The Long Road to Universalism? Recent Developments in the Financing of Long-Term Care in England" En: Social Policy \& Administration, 44(4), 2010. pp. 375-391; RANCI, C y PAVOLINI, E: "Not All That Glitters Is Gold: Long-Term Care Reforms in the Last Two Decades in Europe" En: Journal of European Social Policy, 25(3), 2015. pp. 270-285.

55 COE, N, SKIRA, M y VAN HOUTVEN, C, "Long-Term Care Insurance: Does Experience Matter?” En: Journal of Health Economics, 40, 2015. pp. 122-131.

56 BROWN J y FINKELSTEIN, A: "Why Is the Market for Long-Term Care Insurance so Small?” En: Journal of Public Economics, 91(10), 2007. pp. 1967-1991; BROWN J y FINKELSTEIN, A: "Insuring Long-Term Care in the United States". En: The Journal of Economic Perspectives, 25(4), 2011. pp. 119-141. 
El segundo punto consiste en la apertura de una discusión conceptual sobre el derecho a ser cuidado. Reforma la lógica tradicional, sesgada por género, sobre el cuidado como una responsabilidad intrafamiliar, por una lógica de responsabilidad política del Estado ${ }^{57}$. Se reconocen así estas actividades, como trabajo generador de valor y, por tanto, como actividad económica formal. Las consecuencias son amplias, pero una de las que aquí cabe señalar es la correspondencia que este cambio tiene en la legislación. Chile puede transformarse en una de las naciones pioneras en incluir el derecho al cuidado, no solo de menores y personas con discapacidad, sino de todas las personas que los necesiten. En este sentido, los pilares de la Política Social de la UE pueden ser una orientación ${ }^{58}$.

En cualquier caso, se debe tener presente que la tarea solo comienza con la declaración de principios constitucionales y que serán las leyes y decretos, y la forma y tamaño del financiamiento, los que establezcan qué tipo de modelo de atención que desarrollará el país ${ }^{59}$.

En línea con esto y para finalizar, es determinante tener presente la importancia de cómo se diseñe el modelo. Los servicios que tendrá, el mecanismo de financiamiento, la forma de provisión, los prestadores, el copago, etc. Los beneficios y la eficiencia que ha demostrado tener este tipo de políticas pueden menguar significativamente, según se diseñe uno u otro modelo. Para ello, hay que tener presente tres cuestiones. La primera es que un buen sistema es caro. Existe poco margen de construir un sistema de protección de calidad si se destinan pocos recursos a ello. En segundo lugar, debe asegurarse la accesibilidad económica a los servicios. Un mecanismo de financiamiento de ahorro individual o de seguros privados fragmentados, puede dejar sin efecto el esfuerzo legislativo de cobertura. En tercer lugar, de aplicarse transferencias económicas (cash-for-care), éstas deben aproximarse al costo de los servicios reales y contar con un mecanismo de fiscalización que compruebe el uso de estos fondos para la atención a la dependencia. Esto último resulta muy relevante en el caso chileno, ya que una transferencia económica para dependencia puede terminar siendo un subsidio contra la pobreza, sin impacto relevante en el mercado de trabajo, en la igualdad de oportunidades de género ni en la

57 COMELIN, A y LEIVA, S: “¿Quién Debe Cuidar a Los Adultos Mayores? Voces de Cuidadoras y Cuidadores En Chile" En: Revista Latinoamericana de Estudios del Trabajo, 23(38), 2019. pp. 149-170.

58 EUROPEAN COMMISSION, 2017, cit.

59 COSTA-FONT, J, COURBAGE, C y SWARTZ, K., cit. 
autonomía de la persona dependiente. Para la superación de la pobreza se necesitan políticas específicas orientadas a este fin. No parece conveniente disfrazar una ayuda con otra, sólo para cumplir. Por ello, la reforma propuesta actualmente en el parlamento como seguro de la dependencia, no parece ser la más apropiada ${ }^{60}$.

En resumen, Chile tiene un gran desafío por delante, pero también una enorme oportunidad. Una nueva Constitución puede hacer saltar el marco del bienestar chileno en casi medio siglo, ponerlo a la cabeza de las reformas y desafíos sociales que se enfrentarán en las próximas décadas y, sobre todo, podrá devolver la confianza en la institucionalidad, la esperanza y la construcción un objetivo futuro común, que es, en definitiva, lo que constituye una sociedad.

\section{BIBLIOGRAFÍA}

ANTUNES, V y MOREIRA, J: “Approaches to Developing Integrated Care in Europe: A Systematic Literature Review" En: Journal of Management \& Marketing in Healthcare, 4(2), 2011. ARANCO, N: "Panorama de Envejecimiento y Atención a La Dependencia: Estado de Salud de América Latina y El Caribe" En: Resumen de Políticas, IDB-PB 273, 2018.

ARANCO, N: Panorama de Envejecimiento y Atención a La Dependencia: Resumen Uruguay. BID, Washington D.C., 2019.

ARCE, P, CERÓN, G, GONZÁlEZ, F, GUERRERO, M y PINTO, S: Caracterización de La Dependencia En Las Personas En Situación de Discapacidad a Partir Del II Estudio Nacional de La Discapacidad. Servicio Nacional de la Discapacidad, Santiago, 2017.

ASOCIACIÓN ESTATAL DE DIRECTORES Y GERENTES DE SERVICIOS SOCIALES: XX Dictamen Del Observatorio de La Ley 39/2006 de Promoción de La Autonomía Personal y Atención a Las Personas En Situación de Dependencia. AEDRSS, Madrid, 2020.

60 MATUS-LÓPEZ, M: "Un Sistema de Atención a La Dependencia Para Chile. Apuntes Desde La Experiencia Española”. En Revista de Economía de la Salud, 1(1), 2020. pp. 54-65. 
BARRIGA, F, SANHUEZA, G, SATO, A y SÁEZ, B.: No Es Amor, Es Trabajo No Pagado Un Análisis Del Trabajo de Las Mujeres En El Chile Actual. Fundación Sol, Santiago de Chile, 2020.

BOENINGER, E: Democracia En Chile. Lecciones Para La Gobernabilidad. Andrés Bello, Santiago de Chile, 1998.

BROWN J y FINKELSTEIN, A: "Insuring Long-Term Care in the United States". En: The Journal of Economic Perspectives, 25(4), 2011.

BROWN J y FINKELSTEIN, A: "Why Is the Market for Long-Term Care Insurance so Small?" En: Journal of Public Economics, 91(10), 2007.

CADEMARTORI, J: Chile. El Modelo Neoliberal. CESOC, Santiago de Chile, 1998.

CARUSO, M, GALIANI, S e IBARRARAN, P: "Long-Term Care in Latin America and the Caribbean: Theory and Policy Considerations" En Economia-Journal of the Latin American and Caribbean Economic Association, 20(1), 2019.

CENTRUM INDICATIESTELLING ZORG, "Feiten En Cijfers" Disponible en: https://www.ciz.nl/over-ciz/feiten-en-cijfers Acceso el 25 de julio de 2020.

CID, C. dir.: Análisis Prospectivo de Un Seguro Obligatorio de Dependencia Para Adultos Mayores. Informe Final. CIEDESS, Santiago de Chile, 2014.

COE, N, SKIRA, M y VAN HOUTVEN, C, "Long-Term Care Insurance: Does Experience Matter?" En: Journal of Health Economics, 40, 2015.

COLOMBO, F et al.: Help Wanted? Providing and Paying for Long-Term Care, OECD Publishing, París, 2011.

COMAS-HERRERA, A, WITTENBERG, R y PICKARD, L: "The Long Road to Universalism? Recent Developments in the Financing of LongTerm Care in England”. En: Social Policy \& Administration, 44(4), 2010.

COMELIN, A y LEIVA, S: “¿Quién Debe Cuidar a Los Adultos Mayores? Voces de Cuidadoras y Cuidadores En Chile" En: Revista Latinoamericana de Estudios del Trabajo, 23(38), 2019.

COSTA-FONT, J, COURBAGE, C y SWARTZ, K: "Financing Long-Term Care: Ex Ante, Ex Post or Both?” En Health Economics, 24(51), 2015. 
COSTA-FONT, J, y VILAPLANA-PRIETO, C: "More than One Red Herring'? Heterogeneous Effects of Ageing on Health Care Utilisation". En Health Economics, (forthcoming), 2020.

COTLEAR, D: Population Aging: Is Latin America Ready? World Bank, Washington D.C., 2011.

DA ROIT, B y GORI, C: "The Transformation of Cash for care Schemes in European Long-term Care Policies" En: Social Policy \& Administration, 53 (4), 2019.

DA ROIT, B y LE BIHAN, B: "Similar and Yet So Different: Cash-for-Care in Six European Countries' Long-Term Care Policies". En: The Milbank Quarterly, 88(3), 2010.

DA ROIT, B: "Long-Term Care Reforms in the Netherlands". En: Ranci, Costanzo y Pavolini, Emmanuele: Reforms in Long-Term Care Policies in Europe. Springer, New York, 2013.

DE MEIJER, C, DE MEIJER, C, KOOPMANSCHAP, M, BAGO D'UVA, T y VAN DOORSLAER, E: "Determinants of Long-Term Care Spending: Age, Time to Death or Disability?". En Journal of Health Economics, 30(2), 2011.

DIJKHOFF, T: "Long-Term Care in the Netherlands". En: Becker, Ulrich y Reinhard, Hans-Joachim: Long-Term Care in Europe: A Juridical Approach: Springer International Publishing, Cham, 2018.

EUROPEAN COMMISSION, "The 2018 Ageing Report: Economic and Budgetary Projections for the 28 EU Member States (2016-2070)" En Institutional Paper, 79, 2018.

EUROPEAN COMMISSION: "The 2015 Ageing Report: Economic and Budgetary Projections for the 28 EU Member States (2013-2060)". En European Economy, 3, 2015.

EUROPEAN COMMISSION: European Pillar of Social Rights. EC, Bruselas, 2017.

FINSCHI, A: “Adulto Mayor y El Derecho Chileno. Estado Actual En Chile, Análisis Jurídico Comparado y Observaciones a La Convención Interamericana de Derechos Humanos de Las Personas Mayores" En: Revista Familia y Derecho, 1, 2018. 
GANGA, F, PIÑONES, M, GONZÁLEZ, D y REBAGLIATI, F:: "Rol Del Estado Frente Al Envejecimiento de La Población: El Caso de Chile". En Convergencia, 22(71), 2016.

GONZÁLEZ, F, MASSAD, C y LAVANDEROS, F: Estudio Nacional de La Dependencia En Las Personas Mayores, Servicio Nacional del Adulto Mayor, Santiago de Chile, 2010.

GONZÁLEZ, J: El Efecto de La Ambigüedad En La Transición Chilena, RIL Editores, Santiago de Chile, 2008.

GONZÁLVEZ, H: "Género, Cuidados y Vejez: Mujeres en El Medio Del Trabajo Remunerado y Del Trabajo de Cuidado En Santiago de Chile". En Prisma Social: Revista de investigación social, 21, 2018.

HOHNERLEIN, EM: "Long-Term Care Benefits and Services in Italy". En: Becker, Ulrich y Reinhard, Hans-Joachim: Long-Term Care in Europe: A Juridical Approach, Springer International Publishing, Cham, 2018.

HUENCHUAN, S: Envejecimiento, Derechos Humanos y Politicas Públicas. CEPAL, Santiago de Chile, 2009.

JARA MALEŠ, P, MATUS-LÓPEZ, M y CHAVERRI-CARVAJAL, A: "Tendencias y Desafíos Para Conformar Un Sistema de Cuidados de Larga Duración En Costa Rica," Nota Tecnica IDB_TN 1878, 2020.

JOCELYN-HOLT, A: El Chile Perplejo. Del Avanzar Sin Transar Al Transar Sin Parar. Planeta, Santiago de Chile, 1999.

KELDERS, Y y DE VAAN, K: ESPN Thematic Report on Challenges in Long-Term Care. Netherlands. European Commission, Bruselas, 2018.

KINNEY E y CLARK, B "Provisions for Health and Health Care in the Constitutions of the Countries of the World". En: Cornell International Law Journal, 37(2), 2004.

LATHROP, F: "Protección Jurídica de Los Adultos Mayores En Chile". En: Revista Chilena de Derecho 36 (1), 2009.

MAARSE, J y JEURISSEN, P: "The Policy and Politics of the 2015 LongTerm Care Reform in the Netherlands". En Health Policy, 120(3), 2016.

MAISONNEUVE, C De la y OLIVEIRA, J: A Projection Method for Public Health and Long-Term Care Expenditures, Working Papers OECD Economics Department, 1048, 2013. 
MARTÍNEZ, A: "La Protección Jurídica de Las Personas Mayores Desde La Perspectiva de Los Derechos Humanos". En: Revista de Derecho de la UNED, 17, 2015.

MATUS-LÓPEZ, M y CID, C: "New Long-Term Care Policies in Latin America: The National System of Care in Uruguay". En Journal of the American Medical Directors Association, 17(7), 2016.

MATUS-LÓPEZ, M y CID, C: "Building Long-Term Care Policies in Latin America: New Programs in Chile". En Journal of the American Medical Directors Association, 16(10), 2015.

MATUS-LÓPEZ, M y RODRÍGUEZ-MODROÑO, P: "Presiones de Oferta y Demanda Sobre Políticas Formales de Cuidados En Latinoamérica". En Reforma y Democracia, 60, 2014.

MATUS-LÓPEZ, M: “Thinking about Long-Term Care Policies for Latin America”. En: Salud Colectiva, 11(4), 2015.

MATUS-LÓPEZ, M, y CID, C: “Costo de un sistema de atención de adultos mayores dependientes en Chile, 2012 - 2020". En Pan American Journal of Public Health, 36(1), 2014.

MATUS-LÓPEZ, M, y RAPUN. M: "Los Cuidados Para La Atención a La Dependencia." En Gálvez, Lina dir.: La Economía de Los Cuidados. DeCulturas, Sevilla, 2016.

MATUS-LÓPEZ, M: "Un Sistema de Atención a La Dependencia Para Chile. Apuntes Desde La Experiencia Española". En Revista de Economía de la Salud, 1(1), 2020.

MEDELLIN, N, JARA, P y MATUS-LOPEZ, M: "Envejecimiento y Atención a La Dependencia En Costa Rica," Nota Técnica IDB-TN-1820, 2019.

MINISTERIO DE DESARROLLO SOCIAL DE URUGUAY: Sistema Nacional de Cuidados. Disponible en: https://www.gub.uy/sistemacuidados. Último acceso el 17 de julio de 2020.

MINISTERIO DE DESARROLLO SOCIAL: Adultos Mayores. Síntesis de Resultados. CASEN 2017, MIDESO, Santiago, 2017. Disponible en: http:// observatorio.ministeriodesarrollosocial.gob.cl/casen-multidimensional/ casen/docs/Resultados_Adulto_Mayores_casen_2017.pdf. Último acceso el 20 de julio de 2020 . 
MINISTERIO DE SANIDAD CONSUMO Y BIENESTAR SOCIAL: Estadistica Mensual SAAD, Disponible en: https://www.imserso.es/ imserso_01/documentacion/estadisticas/info_d/estadisticas/est_inf/inf_gp/ index.htm. Último acceso el 16 de julio de 2020.

MONTOYA, C: La Salud Dividida. Chile 1990-2010, CEIBO Ediciones, Santiago de Chile, 2013.

MOUlian, T: Chile Actual. Anatomía de Un Mito. LOM: Santiago de Chile, 1998.

NORORI, M “Addressing the 'Tsunami' of Long-Term Care Needs in Latin America: Is Preparation Feasible?". En Journal of the American Medical Directors Association, 19(9), 2018.

OECD, Health at a Glance 2013, OECD Publishing, París, 2013.

OECD: Health at a Glance 2019. OECD Publishing, París, 2019.

OLIVI, A, FADDA, G y PIZZI, M "Evaluación de La Calidad de Vida de Los Adultos Mayores En La Ciudad de Valparaíso" En: Papeles de población, 21(84), 2015.

PELÁEZ, E y MINOLDO, S: "Impacto Del Envejecimiento Sobre Demandas de Servicios En El Cono Sur". En Revista Latinoamericana de Población, 23, 2018.

PEÑA-LONGOBARDO, L, OLIVA-MORENO, J, GARCÍA-ARMESTO, S y HERNÁNDEZ-QUEVEDO, C: "The Spanish Long-Term Care System in Transition: Ten Years since the 2006 Dependency Act". En Health Policy, 120(10), 2016.

RANCI, C y PAVOLINI, E: "Not All That Glitters Is Gold: Long-Term Care Reforms in the Last Two Decades in Europe" En: Journal of European Social Policy, 25(3), 2015.

RODRÍGUEZ, J, RUSSO, M y CARRASCO, M: "Políticas Públicas Para Una Población Que Envejece: Panorama y Propuestas Para El Sistema de Salud Chileno". En Temas de la Agenda Pública, 12(92), 2017.

SANFORD, A, ORRELL, M, TOLSON, D, ABBATECOLA, A, ARAI, H. BAUER, J y CRUZ-JENTOFT, A: “An International Definition for 'Nursing Home”. En: Journal of the American Medical Directors Association 16(3).

SCHUT, F y VAN DEN BERG, B: "Long-Term Care Insurance in the Netherlands". En Costa-Font, J. y Courbage, C. (Eds.): Financing 
Long-Term Care in Europe: Institutions, Markets and Models. Palgrave Macmillan, Hapshire, 2011.

SOTTOLI, S: "La Política Social En América Latina Bajo El Signo de La Economía de Mercado y La Democracia" En: European Review of Latin American and Caribbean Studies, 68, 2000.

UNITED NATIONS: "Revision of World Population Prospects". Disponible en: https://population.un.org/wpp. Último acceso el 15 de julio de 2020.

VERBEEK-OUDIJK, D WOITTIEZ, I, EGGINK, R y PUTMAN, L: Who Cares in Europe? A Comparison of Long-Term Care for the over-50's in Sixteen European Countries, Netherlands Institute for Social Research, The Hague, 2014.

VILAPLANA, C: "Estimation of Dependency Situations in Spain Using the EDAD 2008”. En Hacienda Pública Española, 194, 2010.

VILLALOBOS, P: "Long-Term Care Systems as Social Security: The Case of Chile". En Health Policy and Planning, 33(9), 2018.

VILlALOBOS, P: "Panorama de La Dependencia En Chile: Avances y Desafíos". En: Revista Medica De Chile, 147(1), 2019.

WEBSTER, CH: The National Health Service: A Political History, Oxford University Press, Oxford, 1998.

WHO: A Glossary of Terms for Community Health Care and Services for Older Persons, World Health Organization, Kobe, 2004.

WU, CH, HUANG, N, FANG, Y, CHOU, Y y Li, CH.: "Determinants of Long-Term Care Services among the Elderly: A Population-Based Study in Taiwan”. En PLoS ONE, 9(2), 2014. 